\title{
Neuer Vertriebschef für die DACH-Region
}

Timo Hand ist seit Jahresanfang Executive Director of Sales für Deutschland, Österreich und die Schweiz beim Dentalhersteller Hu-Friedy. In dieser Position wird er die strategische Ausrichtung in den betreffenden Ländern definieren und das Wachstum forcieren. Zudem übernimmt er die Strukturierung der Geschäftsaktivitäten im europäischen Headquarter in Frankfurt am Main.

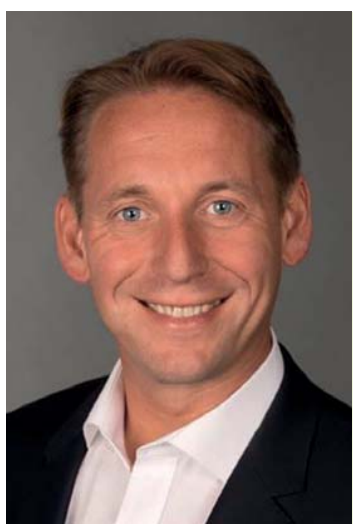

Timo Hand
Timo Hand ist seit 23 Jahren in der Dentalindustrie tätig, zuletzt war er bei ACTEON als Prokurist Mitglied der Geschäftsleitung. Darüber hinaus leitete er den Vertrieb für Deutschland, Nordosteuropa und Zentralasien und zeichnete außerdem verantwortlich für das deutsche Marketing, den Internetauftritt sowie das digitale Kundenmanagement. Der ausgewiesene Experte wird das Unternehmen unter anderem am Standort Frankfurt weiterentwickeln: „Frankfurt befindet sich im Zentrum des europäischen Dentalmarkts. Die hervorragende Infrastruktur und die große Nähe zu Kunden und Lieferanten bieten ideale Voraussetzungen für die Intensivierung der Fachhandelskontakte und die optimale Betreuung von Praxen und Kliniken“ betont Hand.

Nach einer Pressemitteilung der Deutsche Gesellschaft für Zahnärztliche Implantologie e.V., Düsseldorf 